\title{
Protée
}

\section{L’acteur en poseur de signes}

\section{Rodrigue Villeneuve}

Volume 27, numéro 1, 1999

La Mort de Molière et des autres

URI : https://id.erudit.org/iderudit/030534ar

DOI : https://doi.org/10.7202/030534ar

Aller au sommaire du numéro

Éditeur(s)

Département des arts et lettres - Université du Québec à Chicoutimi

ISSN

0300-3523 (imprimé)

1708-2307 (numérique)

Découvrir la revue

\section{Citer cet article}

Villeneuve, R. (1999). L’acteur en poseur de signes. Protée, 27(1), 17-20.

https://doi.org/10.7202/030534ar

\section{Résumé de l'article}

Après avoir rapporté la description habituelle de l'entreprise wilsonnienne comme la production d'un objet lisse ayant la prétention d'échapper au sens, l'auteur montre que cette position, théoriquement, n'est pas tenable et que, pratiquement, elle est combattue par l'exceptionnelle référentialité de La Mort de Molière, de même que par son organisation en récit. Il fait l'hypothèse qu'elle l'est aussi par une autre singularité de l'oeuvre : l'interprétation de Molière par Wilson lui-même. d'utilisation que vous pouvez consulter en ligne.

https://apropos.erudit.org/fr/usagers/politique-dutilisation/ 


\title{
L'ACTEUR EN POSEUR DE SIGNES
}

RODRIGUE ViLLENEUVE

\author{
Mais le sens n'a strictement rien à voir \\ avec l'art, entends-tu, strictement rien! \\ Le sens, c'est de l'assurance sociale qui se prend \\ pour de la métaphysique. \\ Jean-Pierre Vidal, Le Neveu de Stockhausen
}

\section{L'ENVOÛTEMENT ET LE SENS}

L'œuvre de Robert Wilson, l'œuvre théâtrale principalement, mais aussi l'œuvre vidéographique, pose dès le départ à qui veut l'analyser une difficulté importante: elle se donne comme se dérobant au sens. Le spectateur, pour une part de lui-même, si petite s'avouera-t-il qu'elle soit, veut toujours comprendre. Mais le spectacle wilsonnien, si l'on se fie par exemple à la description insistante qu'en donne Frédéric Maurin, n'aurait «rien à dire et tout à montrer. Et depuis la salle, rien à comprendre» ${ }^{1}$. On ne saurait être plus clair. La perfection de l'image, associée à sa répétition, à la lenteur, et à l'absence ou presque de liens narratifs, réduirait en quelque sorte le spectateur au silence. Tout ne serait que regard. Happé par l'image, le spectateur, décrypteur impuissant, sentirait qu'il n'a d'autres choix que de laisser filer, de s'abandonner à la fascination, de «flotter». Mené vers quoi? comment? Qu'importe. Il saurait qu'il n'aura de plaisir qu'en se soumettant. Victime ravie d'une forme éminemment contemporaine de l'illusion théâtrale, il rejoindrait, paradoxalement, ces spectateurs de l'ancien théâtre que fustigeait Brecht.

Maurin décrit fort bien cet effet. Le vide (sémiotique) de l'image wilsonnienne, son "vide scintillant», ferait précisément sa force. Il mettrait en branle l'imaginaire du spectateur et provoquerait une libération fantasmatique. "Je me borne à tisonner le mental assoupi», a déjà déclaré Wilson ${ }^{2}$. En subvertissant le sens, en détruisant la communication, il renverrait le spectateur à lui-même comme à la seule réponse possible à l'énigme que constitue peut-être toujours l'image, en tout cas celle qu'il produit et qui se trouverait dotée ainsi d'une sorte de caractère absolu, toujours intraduisible, toujours irréductible. 
On ne peut pas ne pas penser ici à Mallarmé qui défend, dans "Crayonné au théâtre", l'idée que le théâtre, incité par ce pur espace de suggestion qu'est devenue la scène, ne se réalise pleinement que dans l'esprit du spectateur, qui prend en quelque sorte le relais et fournit à la représentation sa scène ultime, loin de l'immonde bête de carton-pâte qui se pavane derrière la rampe, dans la pauvre lumière du gaz.

La scène comme espace de projection appartient au dispositif inventé par les Grecs. Celui-ci a deux moteurs: la mimésis et la catharsis. Le modèle mallarméen, qui fonctionne à l'affect, comme l'aristotélicien, s'en distingue cependant radicalement en ce qu'il en est un d'appropriation (ou d'ingestion) plutôt que de transfert. La perfection mimétique, réduite à la suggestion et liée à la quasi-disparition de la narrativité, ne provoque pas l'identification mais l'envoûtement. Le sujet ne disparait pas dans l'image, il est activé par elle. Le spectacle ne produit donc plus un message (ou des messages), à traduire avec plus ou moins de facilité, plus ou moins de "vérité», il produit un effet. S'il y a encore sémiotisation, celle-ci n'est plus de l'ordre de la production d'un objet de connaissance, équivalent temporaire et partiel du spectacle, mais de l'ordre de ce que j'ai appellé la confidence: la production d'un objet autre, littéraire, voire spectaculaire, possédant son autonomie propre, obéissant à ses règles, procédant à son autovérification. Ce sera, par exemple, Sarah Bernhard devenue la Berma dans À la Recherche $d u$ temps perdu de Proust ou l'Argentina dansée par Kasuo $\mathrm{Ono}^{3}$. Mais il arrivera le plus souvent qu'il n'y ait qu'un effet, plus ou moins puissant, à l'aune duquel on jugera la qualité du spectacle. Le spectateur comblé est par définition médusé. Il n'a pas à dire, encore moins à faire.

Ce qui peut sembler étonnant, c'est que par des moyens opposés, Wilson produise le résultat dont Mallarmé rêvait. L'affirmation soulignée de l'image scénique est l'équivalent de son quasi-effacement. Pour le reste, même délitescence du récit, même importance accordée à la lumière et à la musique, même place centrale faite au geste. De ce point de vue, il est difficile de ne pas voir dans l'entreprise wilsonnienne un des aboutissements de la modernité théâtrale, qui ne peut s'accomplir, là encore, malgré l'apparent paradoxe, que par la destruction de la représentation. En effet, Wilson, en évidant l'image, en la dépouillant de sa référentialité, comme Mallarmé, la libère, l'«ectoplasmise».

\section{LA PLACE DE L'H(H)ISTOIRE}

Bien des attitudes sont possibles face à cette réalité spectaculaire. On peut y voir une forme hautement sophistiquée du divertissement contemporain, le bel objet lisse, indifférent au monde, indifférent au sens, s'inscrivant parfaitement dans cette standardisation du regard produite par les sociétés technologiquement les plus avancées.

Il y a effectivement quelque chose de cet ordre dans La Mort de Molière. Elle est bien ce produit culturel de très haute qualité, auquel les qualificatifs d'étrange et de fascinant pourront encore une fois être appliqués. On peut donc - c'est l'attitude attendue - y trouver l'occasion d'excitantes dérives fantasmatiques. On peut aussi, agacé, le refuser. Ou s'en sentir exclu. Ou simplement s'y ennuyer.

La question de ce que l'on pourrait maladroitement appeler la valeur éthique ou idéologique d'une telle production n'est évidemment pas notre affaire, bien qu'il y ait là une riche matière à discussion. Ce qui nous intéresse, c'est d'abord de préciser la position dans laquelle se trouve celui qui veut, ou doit, faire sens. L'objet lui dit, de par son dispositif, qu'il n'a rien à faire là. Il se retrouve, gêné, parmi les mauvais spectateurs. Mais l'attitude sémiotique est-elle bien incompatible avec l'objet wilsonnien? N'y a-t-il vraiment rien à comprendre et tout à éprouver, de sorte que tout discours d'analyse est dès le départ posé comme inadéquat et inopérant?

Cette position ne me semble pas tenable. On ne peut poser comme un a priori l'impossibilité de la sémiotisation du monde. Si tout est à éprouver, tout est signe aussi. En même temps. Il n'en va pas de manière différente pour l'œuvre d'art, quelle qu'en soit l'intentionnalité ou la forme. En éprouver les 
effets (plaisir intense, ennui, dégoût, etc.) ne délivre jamais de l'obligation du sens. Entendons-le ici, dans le champ des productions esthétiques, de manière large, comme l'apparition de ce qu'Eco appelle l'idiolecte de l'œuvre: sa structure, son code propre, sa cohérence, construction tout aussi nécessaire que temporaire.

Par ailleurs, ce que la critique wilsonnienne applique à l'ensemble de l'œuvre, à savoir que l'image se contente de mettre en forme sans rien exprimer (Maurin), ne me semble plus convenir aussi bien à $L a$ Mort de Molière. Non pas qu'on n'y retrouve tous les procédés chers à Wilson, mais ils se rapportent ici à un sujet historique. Ce n'est pas la première fois. Les figures de Freud, Einstein, Staline, Rudolf Hess, parmi bien d'autres, traversent l'œuvre de Wilson. Mais jamais le référentiel n'a été aussi précis, ni aussi développé: on se trouve ici en présence d'une représentation relativement claire d'un moment (l'agonie et la mort) de la vie d'un personnage célèbre (Molière).

De plus cette référentialité s'organise comme un récit, avec ses ellipses bien sûr, ses étranges ruptures, ses reprises, ses retours en arrière, mais appuyé quand même sur un développement chronologique dont les principales étapes sont assez facilement repérables.

La Mort de Molière dit donc quelque chose: elle ne fait pas que montrer. Elle renvoie à quelque chose qui lui est extérieur et qui n'est pas d'abord l'imaginaire du spectateur mais son encyclopédie. Il y a donc là une première couche de sens. Il importerait de prendre la mesure de cette part référentielle, ne seraitce que pour voir comment elle est posée là comme un leurre, désavouée, détournée par la manière de montrer. L'essentiel, sans doute, sera finalement étranger à Molière, à ce montage de ses biographèmes. Mais le chemin parcouru pour cerner ce centre (sens) incertain passe si fortement par lui que ce ne saurait être impunément. À l'origine déjà, il y a le tableau représentant la mort du dramaturge accroché au mur du bureau de l'Administrateur de la ComédieFrançaise et qui a servi de point de départ à la vidéo de Wilson. Celle-ci n'y fait pas la moindre allusion. Elle n'en reste pas moins le «travail» de ce tableau laissé hors-champ, son expansion contemporaine dans l'espace et le temps. Bref, s'il est vrai qu'on peut dénoncer chez Wilson «un nivellement des composantes de la mémoire culturelle» ${ }^{4}$, on pourrait facilement vérifier que, dans La Mort de Molière, la référence résiste et vient contrarier la libération fantasmatique que produirait la seule beauté de l'image, ou la musique répétitive de Glass, ou la dérive des citations.

\section{JOUER MOLIĖRE}

La vidéo présente aussi une autre singularité plus difficile à cerner. Elle est, a-t-on le sentiment, traversée par ce qu'on pourrait appeler une inquiétude qui vient ébranler sa surface apparemment si lisse. Cette inquiétude est sans doute liée au sujet lui-même, l'agonie, porteur attendu de morbidité. Mais on se rend compte assez rapidement qu'elle se développe à travers un surprenant réseau de superpositions, dont le spectateur prend peu à peu conscience. Ainsi Heiner Müller, dramaturge gravement malade, écrit sur Molière, dramaturge agonisant. Ce rapprochement du «héros» et de l'énonciateur se double d'une autre, encore plus serrée, qui consiste à faire jouer Molière par Robert Wilson, le réalisateur de la vidéo. Et que trouve-t-on juste au milieu de La Mort de Molière? La lecture d'une longue lettre d'un ami de Robert Wilson, Edwin Denby, qui commence par ces mots: «Tu dois soigner ta toux Bob» et qui, étrangement partagée entre la tendresse amicale et l'ironie, est tout entière consacrée à cette indisposition. La série s'achève ainsi par le renvoi que ne peut manquer de faire le spectateur un peu averti au topos moliéresque par excellence: Molière se mourant, jouant en toussant Argan du Malade imaginaire.

Il est difficile de ne pas tenir compte d'un tel poids aussi bien indiciel que mimétique. Si l'image renvoyant sans cesse à elle-même et aux autres images peut effectivement provoquer chez Wilson le sentiment d'un vide vertigineux, on est bien obligé de constater qu'ici, dans le cas de la représentation de Molière lui-même, tout s'opacifie au contraire 
Et c'est ainsi qu'on est renvoyé à l'acteur Wilson comme au cour de cette oeuvre. Comment, par exemple, dans ce cadre où tout est contrôlé et soigneusement fixé interpréter ce qu'on pourrait appeler son pathos? Car si d'ordinaire chez lui l'acteur ne dispose d'aucune liberté en tant qu'interprète, on sent bien que lui-même se laisse aller dans le rôle de Molière a une réelle liberté expressive. Que faire de ce plein? Quel sens lui donner?

\section{LE TOMBEAU DU POSEUR DE SIGNES}

On est renvoyé, par le biais opposé, à la manière dont la question du sens se pose dans cette œuvre: ou trop de vide, ou trop de plein. Et peut-être est-il normal que ce soit sur l'acteur que nous butions avec cette manière de poser la question. Rappelons quelques évidences. L'acteur fait sens, évidemment: il dit, montre, communique. L'acteur ne fait pas sens, évidemment: tout ce qui est chez lui de l'ordre de «la corporéité expressive et pulsionnelle " 5 est stimuli et sensation plutôt que signe.

Quand on dit que Wilson joue, on entend donc qu'en tant que corps technique, artificiel, crédible et informatif (Barba), il produit un effet sémiotique (ce qui ne signifie pas pour autant que cet effet soit facilement mesurable). On toucherait là à la dimension davantage "wilsonnienne», en vérité souvent exclusive, du jeu, fondée sur la dématérialisation, la dépsychologisation, l'opérationalisation plutôt que l'expression, et finalement l'abstraction.

Mais on entend aussi qu'il produit un effet «énergétique», comme dirait Lyotard ${ }^{6}$. À notre étonnement, en effet, on croit saisir ici l'expression et l'excès - d'un sujet. En d'autres mots, Wilson se comporterait dans La Mort de Molière comme n'importe quel acteur occidental traditionnel! C'est ce fait qui est intéressant et c'est lui qu'il faudrait interroger d'abord, avant de tenter une analyse du jeu dont les risques sont encore aujourd'hui considérables tant sont pauvres les outils dont disposent pour cela les études théâtrales. Il est en tout cas intéressant qu'au corps tenu par l'image, fabriqué par elle, sans autonomie, sans effraction, sans dépassement, qu'au corps dont toute l'énergie semble venir de la caméra, s'oppose et s'ajoute un corps inquiet, vieilli, gémissant, qui ne peut plus être contenu par la «belle image», ni retenu par elle, qui du fauteuil usé est définitivement passé au lit, et qu'on ne verra pas transféré sous la dalle, enfin apaisé.

Serait-il possible qu'ayant renoncé à ce que Kantor appelle la répétition formelle, qui est celle sur laquelle se sont appuyés la modernité américaine (musique, danse, peinture) et le travail de Wilson lui-même, celui-ci soit passé, dans La Mort de Molière, à la répétition métaphysique, qui est ce «sacrilège envers la nature, envers Dieu» que commet tout acteur puisque «je n'ai pas le droit de répéter après la nature, après Dieu " ? ? Le ressassement wilsonnien aurait-il trouvé dans le fait de mourir en Molière une butée?

La visée moderniste rencontre toujours la même résistance lorsqu'il s'agit du théâtre: l'acteur ne peut jamais vraiment s'abstraire, ne peut pas ne pas être et représenter. Wilson ici assumerait totalement ce scandale en jouant lui-même l'Acteur (Molière) et en proposant sa présence propre au déni figuratif de l'art moderne. Mort-vivant, comme tout acteur, il prendrait ainsi le risque que cet exercice rhétorique du tombeau dessine le sien.

\section{NOTES}

1. F. Maurin, $1998: 217$.

2. R. Wilson, cité par F. Maurin, 1998: 224.

3. Voir R. Villeneuve, 1995: 72.

4. F. Maurin, 1998: 70.

5. M. Febvre, 1995: 46.

6. Voir J.-F. Lyotard, 1973.

7. «Kantor présent». Entretien avec Anne Ubersfeld, paru d'abord

dans Théâtre/Public, mai-juin 1981, et repris dans Entretiens, 1996: 59.

\section{RÉFÉRENCES BIBLIOGRAPHIQUES}

BARBA, E. [1993]: «Le Canoë de papier», Bouffonneries, n 28-29. FEBVRE, M. [1995]: Danse contemporaine et théâtralité, Paris, Éd. Chiron. Kantor, T. [1996]: Entretiens, Paris, Éd. Carré.

KLinkenberg, J.-M. [1996] : Précis de sémiotique générale, Bruxelles, De Boeck Université.

LYOTARD, J.-F. [1973]: «La dent et la paume», Des Dispositifs pulsionnels, Paris, UGE.

MAlLARMÉ, S. [1945]: «Crayonné au théâtre », Euvres complètes, Paris, Gallimard, coll. «Bibliothèque de la Pléiade».

MAURIN, F. [1998] : Robert Wilson. Le temps pour voir, l'espace pour écouter, Arles, Actes Sud.

PAVIS, P. [1996]: L'Analyse de spectacles, Paris, Nathan.

VILLENEUVE, R. [1995]: « La photographie du théâtre: les images

échouent toujours", L'Annuaire théâtral, n ${ }^{\circ}$, printemps. 